\title{
环视图表示下的无监督三维物体检索
}

\author{
匡振中，杨结，俞俊 \\ (杭州电子科技大学计算机学院 杭州 310018) \\ (zzkuang@hdu.edu.cn)
}

\begin{abstract}
摘 要: 为了解决基于多视图的三维物体检索方法过度依赖基于人工标注的有监督训练的问题，提出了一种基于环 视图的无监督三维物体检索算法. 首先, 训练面向多圈环视图的无监督深度网络模型, 通过随机数据混合增强学习 不同形状之间的内在联系; 其次，基于最优匹配方法计算物体间的相似性，其中，最优匹配是利用 2 个物体环视图间 最小距离的平均值计算得到; 最后, 利用环视图特征过滤算法去除圥余数据, 能够在保持精度稳定的情况下, 有效 地减少相似性匹配的计算代价. 在 ModelNet40 数据集和 SHREC15 数据集上进行实验, 文中方法精度指标 mAP 分别 为 $41.2 \%$ 和 $54.5 \%$. 实验结果表明, 该无监督三维物体检索方法取得了优异的性能, 有效地降低了人工标注的成本.
\end{abstract}

关键词: 三维物体检索; 无监督; 环视图; 深度学习; 随机混合

中图法分类号: TP391.41 DOI: 10.3724/SP.J.1089.2021.18636

\section{Unsupervised 3D Object Retrieval in Loop View}

Kuang Zhenzhong, Yang Jie, and Yu Jun

(School of Computer Science and Technology, Hangzhou Dianzi University, Hangzhou 310018)

\begin{abstract}
For 3D object retrieval, traditional multi-view methods usually rely on supervised classification for feature learning, which requires lots of manual efforts to annotate data. Differently, we present an unsupervised scheme to deal with the problem by using loop view data. First, we perform unsupervised deep learning of 3D objects for multiple loop view data and we leverage random data mixture to learn the latent relations between different shapes. Then, we introduce an optimal matching method for similarity matching, where the optimal value is calculated by averaging the minimized loop view distances. Finally, we propose a filtering algorithm for loop view features to reduce data redundancy, which can significantly save the computational cost yet preserving the retrieval accuracy. We carry out experiments on two public datasets ModelNet40 and SHREC15. The experimental results show that, compared with related methods, our algorithm has achieved excellent performance for unsupervised 3D object retrieval without requiring data annotation.
\end{abstract}

Key words: 3D object retrieval; unsupervised; loop views; deep learning; random mixture

随着三维扫描技术的飞速发展，三维模型的 识别与检索受到了学术界以及工业界的广泛关注. 三维数据具有多种存储表现形式，如点云、多视
图、多边形网格和体素等. 由于体素和网格数据具 有较高的复杂性和稀疏性，近年来点云数据和多视 图数据受到较多关注. 然而, 这些研究往往过度依

收稿日期：2020-09-03; 修回日期：2020-11-12. 基金项目：国家自然科学基金(61806063，61902101，61802100，61772161). 匡振中(1987一), 男, 博士, 副研究员, 硕士生导师, CCF 会员, 主要研究方向为图像识别、三维模型检索、隐私保护; 杨结(1994一), 男, 硕士研究生, 主要研究方向为图像识别、三维模型检索; 俞俊(1980-), 男, 博士, 教授, 博士生导师, CCF 会员, 主要研究方向为 计算机动画图像处理. 
赖于人工标注, 需要耗费巨大的人力和物力成本.

为了解决上述问题，在多视图识别检索的基 础上，探索了三维物体无监督方式的检索算法. 首 先, 为每个三维模型生成一组环视图, 每圈环视图 图片序列被视为单个训练样本, 分配一个唯一的 序号 id 作为标签. 这样做既可以避免大量的人工 标注任务, 又可以让网络根据三维模型自身的形 状关系去学习. 其次, 使用环视图随机混合的策 略, 可有效地增强模型的性能. 接着, 讨论了 3 种 不同的深度环视图特征混合方式, 分别为最大池 化法、平均池化法和最优匹配法. 最后提出了环视 图特征过滤算法, 在确保精度几乎不变的情况下, 有效地减少了相似性匹配所消耗的计算成本.

\section{1 相关工作}

近年来，随着无人驾驶、虚拟现实技术的飞速 发展, 三维物体检索与识别受到了学术界和工业 界越来越多的关注, 相应地提出了多种方法, 均有 效地改善了三维对象识别与检索的性能. 下面将从 3 个角度依次介绍三维物体识别与检索的方法.

\section{1 基于体素的方法}

该类方法通常使用体素化方法, 将三维物体 表征为一个二值的三维张量, 并通过深度学习模 型来学习涉及的二维变量之间的相关模式. $\mathrm{Wu}$ 等 ${ }^{[1]}$ 最先提出了基于体素的神经网络框架 3D ShapeNets, 用体素网格上二元变量的概率分布来表征 三维物体, 再利用三维卷积网络训练与学习特征. 基于 3D ShapeNets, Maturana 等 ${ }^{[2]}$ 提出了 VoxNet, 为每个输人实例创建多个副本, 并将它们绕轴旋 转一周, 解决了 VoxNet 没有预定义的旋转不变特 征的问题. Sedaghat 等 ${ }^{[3]}$ 使用面向对象的概念对 VoxNet 进行改进，提出了 ORION 模型，不仅可以 预测物体的类别标签，还可以估计物体的方向. Qi 等 ${ }^{[4]}$ 通过一种体素表现形式来学习全局特征, 使用 最大池化来聚合不同方向获得的信息. 为了加快 特征训练, Wang 等 ${ }^{[5]}$ 提出了八叉树数据结构来学 习全局特征. 尽管体素化方法取得了不错的效果, 但仍存在以下缺陷：(1) 局部数据的稀疏性会给特 征提取造成一定影响. $\mathrm{Li}$ 等 ${ }^{[6]}$ 提出了缓解稀疏性问 题的解决方法, 但相关方法的效果依然没有直接 处理点云数据好. (2) 体素化处理过后使用的三维 卷积, 大大增加了训练时的计算量.

\section{2 基于点云的方法}

点云是三维物体在空间中的另一种表示形式,
它的本质是从三维物体表面采集的一系列点. 因 为点云数据具有无序性的特点, 所以传统的深度 学习方法难以直接对其进行处理. Qi 等 ${ }^{[7]}$ 首先提出 了直接处理点云数据的网络 PointNet, 它可以直接 输人点云数据, 并对其进行识别和分割操作; 它使 用对称函数 Max-Pooling 解决了点云存在的无序性 问题, 但是忽略了对点云局部特征的学习. 为了解 决上述问题, Qi 等 ${ }^{[8]}$ 又提出 PointNet++, 采用一个 分级神经网络来提取局部特征. 每个分层结构由 采样层、分组层和特征提取层组成, 即按照一定规 则对每个点组成一个局部区域, 然后使用 PointNet 对该区域提取特征. 基于点云数据的动态卷积神 经网络(dynamic graph CNN for learning on point clouds, DGCNN) $)^{[9]}$ 通过边缘卷积计算了点与点之 间的局部关系，但它只考虑了点的坐标以及点与 邻域点的距离, 并未考虑相邻点的向量方向. Point2Sequence ${ }^{[10]}$ 采用基于注意力机制的序列到序 列模型去学习三维点云的特征, 有效地提高了点 云分类的性能

\section{3 基于多视图的方法}

基于多视图的方法通常通过投影将三维模型 渲染为一组二维视图, 然后将其输人到深度学习 模型进行训练. $\mathrm{Su}$ 等 ${ }^{[11]}$ 提出了多视图卷积神经网 络(multi-view convolutional neural networks, MVCNN) 将从三维物体不同视角渲染得到的二维视图输人 到超深度卷积神经网络 (very deep convolutional networks for large-scale image recognition, $\mathrm{VGG})^{[12]}$, 并对多个视图的特征进行最大池化操作, 得到了 不错的效果. 分组视图卷积神经网络 (group-view convolutional neural networks, GVCNN $)^{[13]}$ 通过加 权分组融合的方式改进了 MVCNN 的融合方法, 更有效地学习了不同视图之间的空间关系. 多视 图双线性池化卷积神经网络 (multi-view harmonized bilinear network for 3D object recognition, $\mathrm{MHBN})^{[14]}$ 通过双线性池化聚集局部卷积特征, 得 到了有效的三维特征表示. 3D2SeqViews ${ }^{[15]}$ 通过 LSTM 和注意力机制, 以序列的方式学习多视图特 征, 取得了不错的效果. 与本文最相似的工作是 $\operatorname{MLVCNN}^{[16]}$, 该方法首次使用二维环视图进行三 维形状识别. 虽然 MLVCNN 改进了传统的三维形 状检索方法, 但并没有充分挖掘环视图中隐藏的 丰富信息用于特征学习. 因为在三维物体检索中 基于无监督的研究比较少, 只有部分基于对抗生 成网络的方法 ${ }^{[17-19]}$, 它们的关注点与本文不同, 所 以未做细致讨论. 


\section{2 本文方法}

本文方法总体流程如图 1 所示. 给定一个三维 模型, 首先生成一系列的环视图数据, 然后将不同 类别的环视图数据随机混合输人到改进后的 GVCNN 模型中进行训练; 接着使用最大池化法、 平均池化法和最优匹配法处理多圈环视图特征, 进行相似性匹配; 最后通过环视图特征过滤算法 过滤出重要性最高的环视图特征, 在保证检索精 度稳定的同时, 有效地降低多圈环视图带来的巨 大计算量.

\section{1 深度环视图的构造}

人类在观察一个未知物体时, 往往绕其一周
观察便可确认它的类别. 绕其一周观察, 仍不知道 它的类别时, 往往是因为选取的绕行角度不好. 这 时会选择以不同的绕行角度去绕行观察多圈, 以 得到更全面的类别特征. 受此启发, 如图 2 所示, 本文将三维物体放置在一个包围球的中心, 在球 面上任选一点将其与球心连接得到一条半径, 再 任意选取一个方向使该半径围绕球心旋转 $360^{\circ}$, 每隔 $30^{\circ}$ 采集一次图片, 称之为一圈环视图. 依据 该方法旋转多圈便可得到多圈环视图, 进而得到 本文需要的环视图数据集. 该数据投影方法有以 下优点: (1) 不需要像传统的投影方法那样, 固定 三维物体的位置和旋转方向. (2) 多圈环视图获取 了更全面的视图特征.

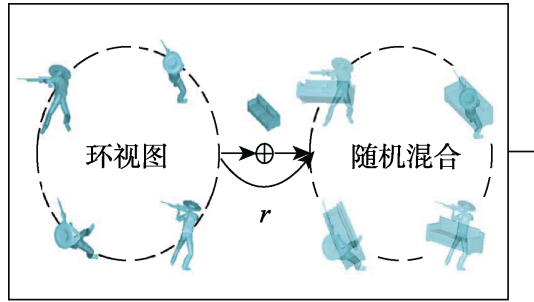

a. 三维数据表征: 混合环视图

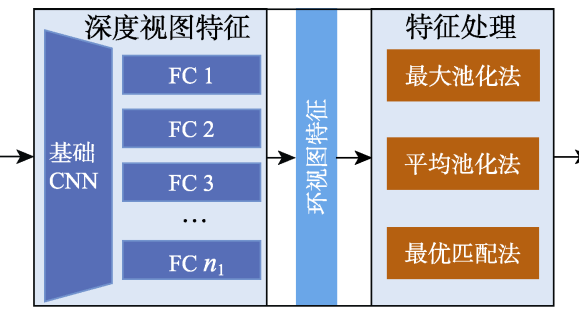

b. 特征学习与提取

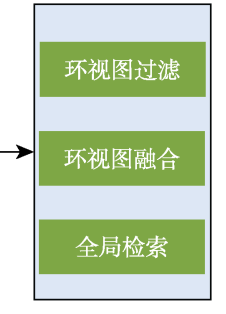

c. 检索

图 1 本文方法总体流程图

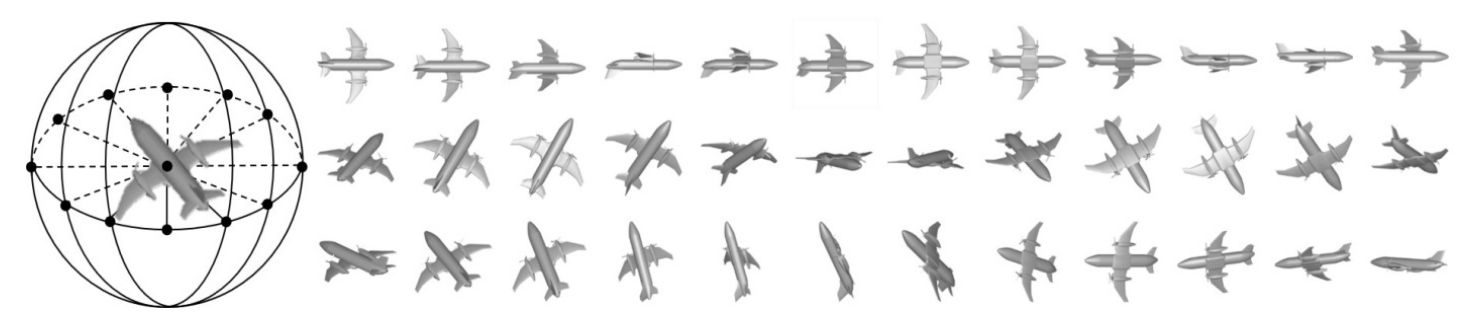

图 2 环视图采集示意图

给定一个无标注 的三维物 体数 据集 $S=\left\{x_{1}, x_{2}, x_{3}, \cdots, x_{n}\right\}$. 给每一个三维物体随机分配 一个唯一的序号 $\operatorname{id}($ 如从 $1 \sim n$ ); 然后将序号 id 作为 物体的语义标签 $y=\left\{y_{1}, y_{2}, y_{3}, \cdots, y_{n}\right\}$. 通过为每一 个三维模型 $x_{i}, i \in\{1,2,3, \cdots, n\}$ 生成如图 2 所示 $n_{1}$ 圈环视图, 这些环视图构成了深度环视图数据

$$
D=\left\{\left(v_{i j}, y_{i}\right), 1 \leqslant i \leqslant n, 1 \leqslant j \leqslant 12 n_{1}\right\}
$$

三维模型 $x_{i}$ 的视图集表示为

$$
V\left(x_{i}\right)=\left\{v_{i j}, 1 \leqslant j \leqslant 12 n_{1}\right\}, 1 \leqslant i \leqslant n
$$

其中, $v_{i j}$ 表示模型 $i$ 的第 $j$ 个环视图数据. 需要特 别指出的是，设定的标签 $y$ 不包含任何类别信息， 从相同的模型获取的环视图共享同一个形状级别 的标签. 在数据采集过程中, 本文随机选择虚拟摄 像机的位置来捕获每圈的环视图, 没有对三维模
型的位置和方向提出任何要求.

\section{2 环视图的随机混合}

近年来，学术界出现了很多基于数据混合的 模型增强方法 ${ }^{[20-22]}$, 显示出数据混合具有良好的 模型增强功能. 本文通过使用环视图数据随机混 合法(random mixture, RM)来扩展这一方法, 使模 型更好地学习不同类之间的特征联系. 对于一圈 环视图 $v \in V\left(x_{i}\right)\left(v\right.$ 表示 $x_{i}$ 多圈环视图中的一圈数 据, 包含 12 张视图). 随机从数据集 $D$ 中选取另一 圈环视图 $u \in V\left(x_{j}\right), i \neq j$. 然后通过以下公式更新 训练样本 $v$ 和标签 $y_{v}$.

2 圈环视图混合后的样本为

$$
v^{\prime}=\left(v_{1}^{\prime}, v_{2}^{\prime}, v_{3}^{\prime}, \cdots, v_{k}^{\prime}, \cdots, v_{12}^{\prime}\right)
$$

其中, 


$$
v_{k}^{\prime}= \begin{cases}v_{k}, & \text { if } r<0.5 \\ \frac{r \cdot v_{k}+(1-r) \cdot u_{k}}{\sqrt{r^{2}+(1-r)^{2}}}, & \text { otherwise }\end{cases}
$$

是 2 张对应单视图的混合视图; $u_{k}, v_{k}$ 表示 $u$ 和 $v$ 混合样本的对应单视图;

$$
y_{v}^{\prime}=r \cdot y_{v}+(1-r) \cdot y_{u}
$$

$r$ 表示生成的随机系数, $r \in(0,1) . r$ 有 2 个作用: (1) 确定是否进行样本混合. (2) 确定样本混合时 使用的比例. $k$ 表示一圈环视图的不同视图， $k=\{1,2,3, \cdots, 12\}$. 最后, 利用 KL 散度损失

$$
D_{\mathrm{KL}}(P \| Q)=\sum_{i=1}^{n} P\left(x_{i}\right) \cdot \ln \left(\frac{P\left(x_{i}\right)}{Q\left(x_{i}\right)}\right)
$$

进行训练. 其中, $P\left(x_{i}\right)$ 表示样本真实的概率分布; $Q\left(x_{i}\right)$ 表示样本预测的概率分布. 随机混合算法将 不同类型的三维模型进行混合后进行训练, 其本 质是一种数据增强行为. 虽然混合数据的分类难 度增加, 但是可以提高模型对数据的拟合能力.

\section{3 最优匹配}

利用训练得到的模型对每一个三维物体提取 环视图特征，这些环视图以联合或者独立的方式 计算相互之间的相似性. 这里有 3 种可以选择的方 法, 分别是最优匹配法(optimal matching, OP)、最 大池化法和平均池化法. 对于最优匹配法, 定义环 视图 $v$ 和 $u$ 之间的距离和环视图 $v$ 和三维物体 $s \in S$ 的距离分别为

$$
\begin{aligned}
& d_{1}(v, u)=\|\varphi(v)-\varphi(u)\|_{2} \\
& d_{2}(v, s)=\min _{u \in V(s)} d_{1}(v, u)
\end{aligned}
$$

其中, $\varphi(v)$ 表示 $v$ 的特征. 给定 2 个三维物体 $s_{1} \in S$
和 $s_{2} \in S$, 定义其最优匹配计算公式为

$$
d_{3}\left(s_{1}, s_{2}\right)=\frac{1}{2 n_{1}}\left(\sum_{v \in V\left(s_{1}\right)} d_{2}\left(v, s_{2}\right)+\sum_{u \in V\left(s_{2}\right)} d_{2}\left(u, s_{1}\right)\right)
$$

最大池化和平均池化将同一三维模型的多圈 数据转化为一个全局特征, 通过欧几里得度量计 算各个模型之间的距离矩阵, 再进行相似性匹配. 显然, 上述操作忽略掉了很多细节信息, 不能够保 证检索的精度.

最优匹配法需要计算大量的环视图特征之间 的欧几里得距离, 耗费了极大的计算资源, 而且存 储相似性距离矩阵也需要占用大量的内存空间. 为了解决上述问题, 本文提出了环视图特征过滤 算法, 并利用注意力机制进行特征融合.

\section{4 环视图特征过滤与融合}

如图 3 所示, 环视图特征过滤网络是一个包含 了卷积层、全连接层、标准化层、特征融合层、环 视图特征过滤层和 Softmax 分类器层的网络. 首先 将提取出的多圈环视图特征输人到环视图特征过 滤网络中, 通过几个全连接层和打分模块后得到 各圈环视图打分; 然后将分值与环视图特征加权 求和, 输人到 Softmax 分类器进行分类训练(这里 的分类依然没有使用具有类别信息的标签, 而是 利用第 2.1 节中提出的序号 id 作为标签). 通过环 视图打分模块可以输出每圈环视图特征的重要性 打分 $\alpha$, 其计算公式为

$$
\alpha=\operatorname{Sigmoid}(\ln (|\theta|))
$$

其中, $\alpha \in(0,1) ; \theta$ 表示 FC4 层的输出. 接着将 FC3 层输出的特征与获得的打分进行加权求和融 合, 最后输人到分类器进行分类. 根据环视图特征 过滤网络，本文提出了 2 种融合方法.

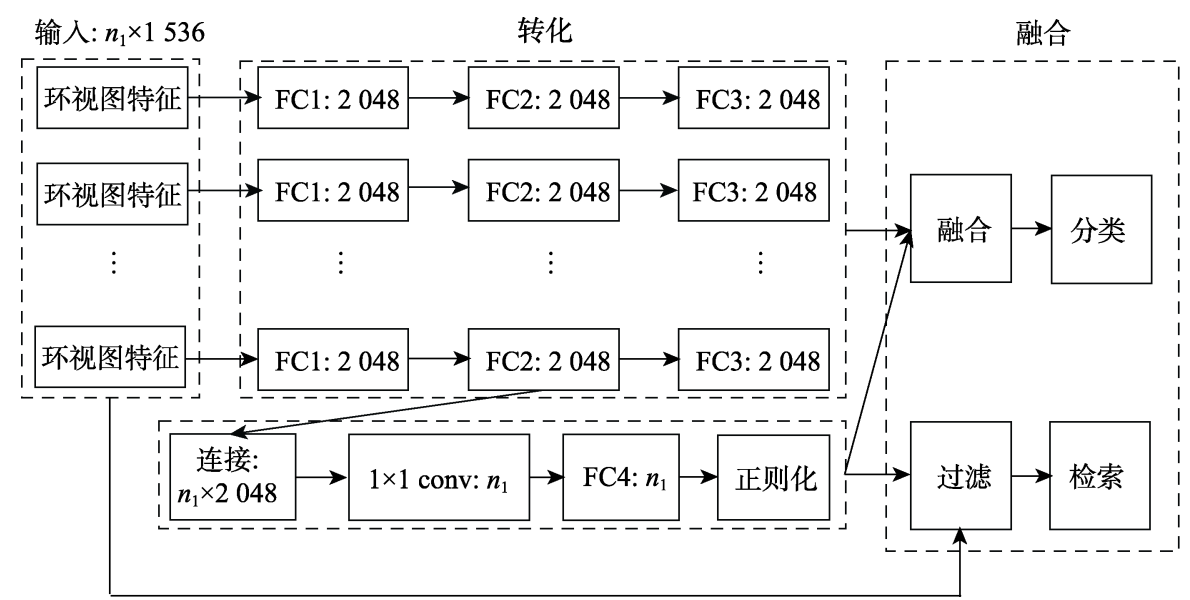

图 3 环视图过滤模块流程图 
(1) 环视图特征过滤法 (attention filter, AF). 依据打分对 FC1 层的输人特征进行升序排序, 然 后依序从大到小选择 $m$ 个特征 $\left(m<n_{1}, n_{1}\right.$ 表示原 有特征的数量); 最后通过式(9)进行相似性匹配.

（2）基于环视图重要性打分融合机制(attention aggregation, AA). 利用已有打分对 FC3 层提取出 来的特征进行加权求和融合, 然后利用式(7)进行 相似性匹配.

这 2 种方法都在保证精度的情况下，有效地加 快了相似性匹配的计算过程.

\section{3 实验与分析}

为了验证方法的有效性，本文分别采用刚性 三维模型数据集 Princeton ModelNet $40^{[1]}$ 和非刚性 三维模型数据集 SHREC15 ${ }^{[23]}$ 进行实验. ModelNet 数据集搜集了 127915 个三维 CAD 模型, 归人 662 个类别; 其中, ModelNet 40 包含 40 个类 12311 个 模型. SHREC15 包含 50 类 1200 个模型. 本文在 ModelNet40 和 SHREC15 上以随机角度分别采集 了 20 圈环视图进行实验. 由于本文方法是完全无 监督的, 故在训练时没有划分测试集与训练集, 训 练与测试都使用所有数据. 最终, 2 种数据集的分 类类别数分别为 12311 和 1200 , 分类精度的最高 结果分别为 $92.9 \%$ 和 $86.7 \%$.

\section{1 最优匹配法}

本节比较了最优匹配法、最大池化法和平均池 化法的性能. 为了公平比较, 这 3 种方法都是在未 加上随机混合和环视图特征过滤的基础上进行实 验. 实验结果如表 1 所示(本文用 R@N 代表检索的 前 $N$ 个三维物体中正确检索物体所占的比例), 可 以观察到: (1) 最大池化法在 2 个数据集上的性能都 要比平均池化法好. (2) 最优匹配法在 2 种数据集上 均优于最大池化法和平均池化法, 尤其在平均检索 精度 (mean average precision, mAP) 指标上, SHREC15 上最优匹配法的 $\mathrm{mAP}$ 为 $47.5 \%$, 而平均 池化法和最大池化法最高为 $41.3 \%$. (3) 3 种方法在 SHREC15 数据集上的检索效果均优于 ModelNet40. 可能是因为 ModelNet40 数据集具有很严重的类间 相似性(如图 4 中的课桌和桌子、沙发和长椅), 加 大了网络的学习难度. 而 SHREC15 数据集具有很 明显的类间差异性，尽管其数据规模小、分类类别 多, 依然取得了比 ModelNet40 数据集更好的效果. 由于最优匹配法取得了最佳的效果，因此下文默 认选择最优匹配法作为相似性度量的基础方法.
表 1 最优匹配、最大池化和平均池化的对比 \%

\begin{tabular}{rccccccc}
\hline 数据集 & 方法 & R@1 & R@5 & R@10 & R@all & mAP \\
\hline SHREC15 & 平均池化法 & 76.5 & 55.1 & 39.1 & 22.3 & 39.4 \\
最优匹配法 & 84.8 & 66.1 & 48.2 & 27.3 & 47.5 \\
\hline 最池化法 & 80.6 & 65.1 & 71.2 & 19.5 & 31.1 \\
\hline 最优匹配法 & 81.4 & 67.3 & 73.1 & 20.6 & 32.3 \\
\hline
\end{tabular}

图 4 检索效果图

\section{2 模型检索方法的验证}

表 2 比较了随机混合法、环视图特征过滤法和 基于注意力机制特征融合法的性能. 其中, $\mathrm{OP} *$ 是使 用单圈环视图特征进行检索的结果. 对比表 2 和表 3 中数据可以发现: (1) OP 的性能远远胜于 OP*, 例如, 在 SHREC15 数据集中, $\mathrm{OP}$ 的 $\mathrm{mAP}$ 为 $47.5 \%$, 而 $\mathrm{OP} *$ 为 $35.2 \%$. 显然, 这样的性能差距是因为多圈环视图 比单圈环视图提供了更多的鉴别性信息. (2) OP+RM 在 OP 的基础上有了显著地提升, 例如, 在 ModelNet40 中, OP+RM的 R@1, R@5, R@10和mAP 在 $\mathrm{OP}$ 的基础上分别提高了 $3.7 \%, 7.0 \%, 8.8 \%$ 和 $8.9 \%$; 可见随机混合法具有良好的模型增强效果. (3) 虽然 最优匹配法和随机混合法具有不错性能, 但是在 ModelNet 40 上对多达 20 圈的环视图进行欧几里得度 量计算和最优匹配计算非常耗时. 如表 3 所示, 单纯 的相似性匹配需耗费 $28.4 \mathrm{~h}$. 本文 2 种融合方法都节 约了大量因相似性匹配而耗费的时间, $\mathrm{OP}+\mathrm{RM}+\mathrm{AA}$ 和 $\mathrm{OP}+\mathrm{RM}+\mathrm{AF}$ 在节省大量计算时间的同时，保证 了精度的稳定. 至于部分指标有所降低也属合理, 因为特征融合在减少计算量的同时丢失了部分有 效信息.

图 4 展示了部分三维模型的检索效果. 图 5 和 图 6 中的 PR(precision recall)曲线展示了本文方法 


\begin{tabular}{clccccc}
\multicolumn{2}{c}{ 表 2 } & 模型检索性能对比 & & $\%$ \\
\hline 数据集 & \multicolumn{1}{c}{ 方法 } & R@1 & R@5 & R@10 & R@all & mAP \\
\hline \multirow{5}{*}{ SHREC15 } & OP* & 74.3 & 49.1 & 34.0 & 19.4 & 35.2 \\
& OP & 84.8 & 66.1 & 48.2 & 27.3 & 47.5 \\
& OP+RM & 95.6 & 78.1 & 55.6 & 30.0 & 54.5 \\
& OP+RM+AF & 96.3 & 77.7 & 55.7 & 30.0 & 54.4 \\
& OP+RM+AA & 94.7 & 74.9 & 53.0 & 28.7 & 52.3 \\
\hline \multirow{5}{*}{ ModelNet40 } & OP* & 78.4 & 69.1 & 62.6 & 18.3 & 28.9 \\
& OP & 81.4 & 73.1 & 67.3 & 20.6 & 32.3 \\
& OP+RM & 85.1 & 80.1 & 76.1 & 26.3 & 41.2 \\
& OP+RM+AF & 85.1 & 79.7 & 75.6 & 25.5 & 40.8 \\
& OP+AA & 85.4 & 80.1 & 76.0 & 26.5 & 40.3 \\
\hline
\end{tabular}

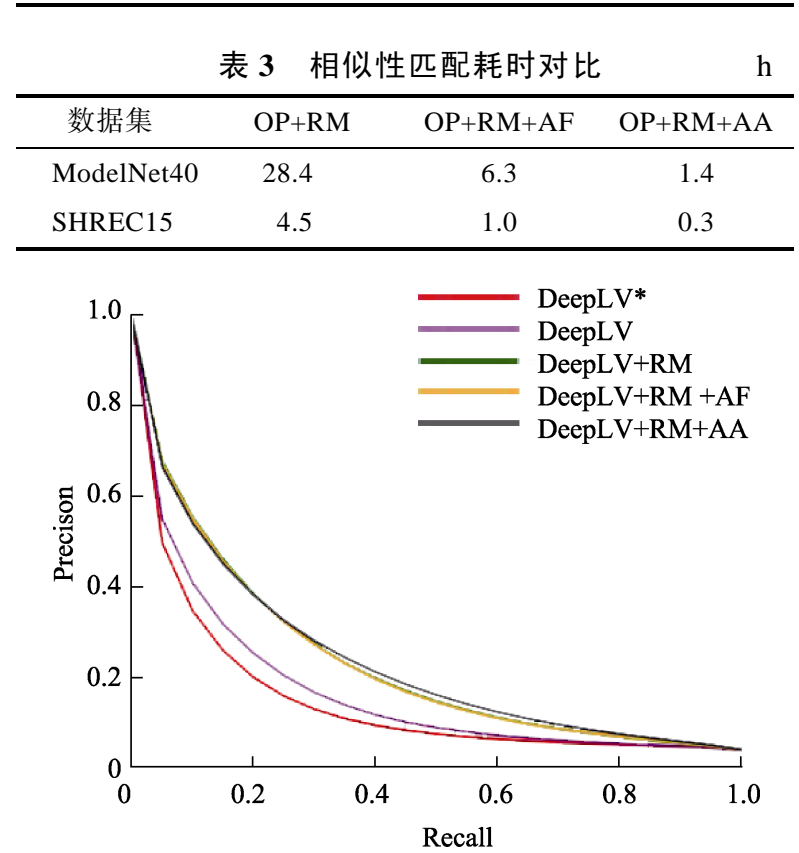

图 5 ModleNet40 PR 曲线图

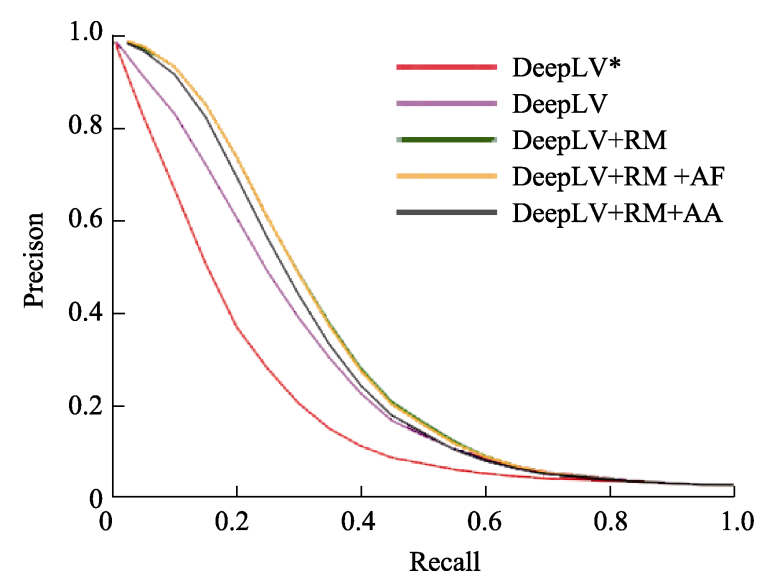

图 6 SHREC15 PR 曲线图

的检索性能; 可视化的结果展示了本文的特征是 基于三维模型本身形状的自然关系去学习，而不 是人工的语义标签. 尽管图 4 中的检索结果有一部 分错误, 但所有的检索结果都有着与输人物体相
似的形状. 由此可以证明, 这些错误的产生主要是 因为三维模型存在类内差异性和类间相似性. 图 4 的最后一行可以清晰地证明这一点, 最后一行输 人课桌, 检索结果为形状类似输人课桌的桌子、钢 琴和椅子.

\section{3 过滤算法的验证}

本节验证了环视图特征过滤算法是否过滤出 了最具有鉴别能力的环视图特征. 实验采用 20 圈 ModelNet40 环视图特征作为输人, 依据打分从小 到大对 20 圈环视图特征进行分组, 总共分为 4 组. 实验结果如表 4 所示, Rank1-5 指打分排序第 1 到 第 5 的特征，对于 Rank6-10, Rank11-15 和 Rank1620 的含义依此类推. 由表 4 可知: (1) 打分最低的 一组即 Rank1-5 具有最差的性能. 可能是因为该组 中包含了很多冗余图片或者渲染角度不好的图片. (2) Rank6-10 和 Rank11-15 这 2 组的性能几乎相同, 而 Rank16-20 有着最佳性能. 这样的结果说明本文 过滤算法过滤出了性能最好的特征. 过滤后的结 果和过滤前依然有一些差距, 这是因为圥余特征 中依然包含一些有效信息, 所以过滤过程中会丢 失部分有效信息. 但是相比之前的计算量, 部分精 度的损失依然可以接受.

表 4 过滤算法性能对比

\begin{tabular}{lccc}
\hline \multicolumn{1}{c}{ 分组 } & R@5 & R@10 & R@all \\
\hline Rank1-5 & 72.1 & 65.8 & 24.6 \\
Rank6-10 & 79.1 & 74.9 & 25.0 \\
Rank11-15 & 79.1 & 74.8 & 25.2 \\
Rank16-20 & 79.7 & 75.6 & 25.5 \\
\hline
\end{tabular}

\section{4 与其他方法的比较}

为了公平地进行比较, 本文在 MVCNN 和 GVCNN 上使用 ModelNet40 数据集进行了无监督 训练, 即按模型序号随机分配 id 作为标签, 进行 12311 分类训练. 需要注意的是, 在训练时没有采 用多圈环视图而是用深度图片集 ${ }^{[24]}$. 实验结果如 表 5 所示, 其中, D 代表深度图数据集. 可见本文 方法在各个性能指标上都优于 MVCNN 和 GVCNN. 其中, 在 mAP 这一指标上比 GVCNN 高 13.4\%, 比 MVCNN 高 $10.0 \%$.

\begin{tabular}{|c|c|c|c|}
\hline 方法 & R@10 & R@All & $\mathrm{mAP}$ \\
\hline GVCNN+D & 65.5 & 16.4 & 27.8 \\
\hline MVCNN+D & 69.1 & 19.3 & 31.2 \\
\hline 本文 & 76.1 & 26.3 & 41.2 \\
\hline
\end{tabular}


与有监督学习算法相比, 本文方法依然有一 定的性能差距. 但当没有数据标签时, 将无法进行 传统的有监督训练, 只能进行无监督训练. 因此, 当缺乏数据标签时，本文方法具有更好的普适性.

\section{4 结 语}

本文在基于多视图的三维物体识别方法的基 础上，提出了一种基于无监督方式的三维物体检 索的方法. 首先利用多圈环视图对三维物体进行 无监督训练, 然后使用随机混合法对模型进行增 强, 接着利用基于环视图特征重要性的融合法和 环视图特征过滤法，对特征进行过滤。最后，在 ModelNet40 和 SHREC15 上分别进行了实验验证. 实验证明，通过本文提出的方法可以取得良好的检 索效果并节省大量的人力物力成本. 但是该方法还 存在一定的缺陷，比如如何解决由类间相似性带来 的影响，让模型更有效地基于物体本身的形状关系 去学习. 这些将在后续的工作中进一步完善.

\section{参考文献(References):}

[1] Wu Z R, Song S R, Khosla A, et al. 3D ShapeNets: a deep representation for volumetric shapes[C] //Proceedings of the IEEE Conference on Computer Vision and Pattern Recognition. Los Alamitos: IEEE Computer Society Press, 2015: 1912-1920

[2] Maturana D, Scherer S. VoxNet: a 3D convolutional neural network for real-time object recognition[C] //Proceedings of the IEEE/RSJ International Conference on Intelligent Robots and Systems. Los Alamitos: IEEE Computer Society Press, 2015: 922-928

[3] Sedaghat N, Zolfaghari M, Amiri E, et al. Orientation-boosted voxel nets for 3D object recognition[OL]. [2020-09-03]. https://arxiv.org/abs/1604.03351

[4] Qi C R, Su H, Nießner M, et al. Volumetric and multi-view CNNs for object classification on 3D data[C] //Proceedings of the IEEE Conference on Computer Vision and Pattern Recognition. Los Alamitos: IEEE Computer Society Press, 2016: 5648-5656

[5] Wang P S, Liu Y, Guo Y X, et al. O-CNN: octree-based convolutional neural networks for 3D shape analysis[J]. ACM Transactions on Graphics, 2017, 36(4): Article No.72

[6] Li Y Y, Pirk S, Su H, et al. FPNN: field probing neural networks for 3D data[OL]. [2020-09-03]. https://arxiv.org/abs/ 1605.06240

[7] Qi C R, Su H, Mo K C, et al. PointNet: deep learning on point sets for 3D classification and segmentation[C] //Proceedings of the IEEE Conference Computer Vision and Pattern Recognition. Los Alamitos: IEEE Computer Society Press, 2017: 77-85

[8] Qi C R, Yi L, Su H, et al. PointNet++: deep hierarchical feature learning on point sets in a metric space[OL]. [2020-09-03]. https://arxiv.org/abs/1706.02413

[9] Wang Y, Sun Y B, Liu Z W, et al. Dynamic graph CNN for learning on point clouds[J]. ACM Transactions on Graphics, 2019, 38(5): Article No.146

[10] Liu X H, Han Z Z, Liu Y S, et al. Point2Sequence: learning the shape representation of $3 \mathrm{D}$ point clouds with an attention-based sequence to sequence network[C] //Proceedings of the AAAI Conference on Artificial Intelligence. Palo Alto: AAAI Press, 2019: 8778-8785

[11] Su H, Maji S, Kalogerakis E, et al. Multi-view convolutional neural networks for 3D shape recognition[C] //Proceedings of the IEEE International Conference on Computer Vision. Los Alamitos: IEEE Computer Society Press, 2015: 945-953

[12] Simnyan K, Zisserman A. Very deep convolutional networks for large-scale image recognition[OL]. [2020-09-03]. https://arxiv.org/abs/1409.1556v1

[13] Feng Y F, Zhang Z Z, Zhao X B, et al. GVCNN: group-view convolutional neural networks for 3D shape recognition[C] //Proceedings of the IEEE/CVF Conference on Computer Vision and Pattern Recognition. Los Alamitos: IEEE Computer Society Press, 2018: 264-272

[14] Yu T, Meng J J, Yuan J S. Multi-view harmonized bilinear network for 3D object recognition[C] //Proceedings of the IEEE/CVF Conference on Computer Vision and Pattern Recognition. Los Alamitos: IEEE Computer Society Press, 2018: 186-194

[15] Han Z Z, Liu H L, Liu Z B, et al. 3D2SeqViews: aggregating sequential views for $3 \mathrm{D}$ global feature learning by CNN with hierarchical attention aggregation[J]. IEEE Transactions on Image Processing, 2019, 28(8): 3986-3999

[16] Jiang J W, Bao D, Chen Z Q, et al. MLVCNN: multi-loop-view convolutional neural network for 3D shape retrieval[C] //Proceedings of the AAAI Conference on Artificial Intelligence. Palo Alto: AAAI Press, 2019: 8513 -8520

[17] Han Z Z, Shang M Y, Liu Y S, et al. View inter-prediction GAN: unsupervised representation learning for 3D shapes by learning global shape memories to support local view predictions[C] //Proceedings of the 33rd AAAI Conference on Artificial Intelligence. Palo Alto: AAAI Press, 2019: 8376-8384

[18] Wu J J, Zhang C K, Xue T F, et al. Learning a probabilistic latent space of object shapes via 3D generative-adversarial modeling[C] //Proceedings of the 30th International Conference on Neural Information Processing Systems. Cambridge: MIT Press, 2016: 82-90

[19] Khan S H, Guo Y L, Hayat M, et al. Unsupervised primitive discovery for improved 3D generative modeling[C] //Proceedings of the IEEE/CVF Conference on Computer Vision and Pattern Recognition. Los Alamitos: IEEE Computer Society Press, 2019: 9739-9748

[20] Tokozume Y, Ushiku Y, Harada T. Learning from between-class examples for deep sound recognition[OL]. [202009-03]. https://arxiv.org/abs/1711.10282v2

[21] Tokozume Y, Ushiku Y, Harada T. Between-class learning for image classification[C] //Proceedings of the IEEE/CVF Conference on Computer Vision and Pattern Recognition. Los Alamitos: IEEE Computer Society Press, 2018: 5486-5494

[22] Zhang H Y, Cisse M, Dauphin Y N, et al. mixup: beyond empirical risk minimization[OL]. [2020-09-03]. https://arxiv.org/ abs/1710.09412

[23] Lian Z, Zhang J, Choi S, et al. Non-rigid 3D shape retrieval[C] //Proceedings of the Eurographics Workshop on 3D Object Retrieval. Aire-la-Ville: Eurographics Association Press, 2015: 107-120

[24] Su J C, Gadelha M, Wang R, et al. A deeper look at 3D shape classifiers[C] //Proceedings of the European Conference on Computer Vision. Heidelberg: Springer, 2018: 645-661 